山्山 FRANÇAISE

$\supset$ DE

필 PEAGOGIE

\section{Revue française de pédagogie}

Recherches en éducation

156 | juillet-septembre 2006

Les espaces locaux d'interdépendance entre

établissements : une comparaison européenne

\title{
LAWN Martin \& GROSVENOR Ian (éd.). Materialities of Schooling : Design, Technology, Objects, Routines
}

Oxford : Symposium Books, 2005. - 217 p. (Comparative Histories of Education)

\section{Anne-Marie Châtelet}

\section{OpenEdition}

\section{Journals}

Édition électronique

URL : http://journals.openedition.org/rfp/681

DOI : $10.4000 /$ rfp.681

ISSN : 2105-2913

Éditeur

ENS Éditions

\section{Édition imprimée}

Date de publication : 1 juillet 2006

Pagination : 186-187

ISBN : 978-2-7342-1060-3

ISSN : 0556-7807

Référence électronique

Anne-Marie Châtelet, « LAwn Martin \& grosvenor lan (éd.). Materialities of Schooling : Design,

Technology, Objects, Routines », Revue française de pédagogie [En ligne], 156 | juillet-septembre 2006,

mis en ligne le 24 septembre 2010, consulté le 25 septembre 2020. URL : http://

journals.openedition.org/rfp/681 ; DOI : https://doi.org/10.4000/rfp.681

Ce document a été généré automatiquement le 25 septembre 2020

(c) tous droits réservés 


\title{
LAWN Martin \& GROSVENOR Ian (éd.). Materialities of Schooling : Design, Technology, Objects, Routines
}

Oxford : Symposium Books, 2005. - 217 p. (Comparative Histories of Education)

\author{
Anne-Marie Châtelet
}

\section{RÉFÉRENCE}

LAWN Martin \& GROSVENOR Ian (éd.). Materialities of Schooling: Design, Technology, Objects, Routines.Oxford : Symposium Books, 2005. - 217 p. (Comparative Histories of Education)

1 À ses premières heures, l'histoire de l'éducation fut celle des doctrines et des méthodes pédagogiques. Avec le temps, certains ont tourné leurs regards vers les expériences d'enseignement, scrutant les comportements des maîtres et des élèves. Ils ont par la suite élargi l'analyse au cadre matériel de l'école. Ce sont des travaux de ce troisième type qu'ont rassemblés Martin Lawn et Ian Grosvenor dans Materialities of Schooling. Tous deux britanniques, professeurs respectivement à l'université de Cardiff et de Birmingham, ils avaient dirigé, avec Kate Rousmaniere, la publicationde Silences and Images: Social History of Classroom ${ }^{1}$. Dans cette première incursion au sein des lieux d'enseignement, ils s'interrogeaient sur l'histoire des salles de classe, au travers de réflexions méthodologiques et de cas d'études, montrant que des sources jusque-là peu exploitées, comme les souvenirs, les correspondances... pourraient être utilisées au service d'une histoire des pratiques de l'enseignement. Ce qu'ils proposent aujourd'hui dans Materialities of Schooling, est de considérer la matière de la classe au sens propre: son cadre et les objets scolaires qui la constituent et qui, généralement ignorés des historiens de l'éducation, doivent leur survie aux conservateurs des musées.

2 L'ouvrage est publié dans une nouvelle collection chez Symposiums Books - et on en trouvera une présentation sur le site internet de l'European Educational Research 
Association, http://www.eera.ac.uk/web/eng/all/networks/network17/report2005/ index.html (consulté le 30 août 2006) - comme l'une de leurs dernières productions, résultant d'un colloque sur le même thème organisé à Lisbonne en 2002 par les auteurs associés à Antonio Nóvóa; cependant rien n'est dit de cette manifestation dans l'ouvrage. Dix auteurs y sont réunis, quatre hispaniques - venus d'Argentine, d'Espagne et du Mexique - cinq britanniques et un Australien, lui donnant un caractère international. Leurs contributions sont présentées en fonction de leur sujet, dans un ordre qui va de ce qui est matériellement le plus visible, l'architecture, à ce qui est plus insaisissable, le temps et l'espace. Chacune est accompagnée d'une bibliographie constituant ainsi au fil des pages, un panorama historiographique intéressant.

L'ensemble débute par un article d'Elsie Rockwell « Murs, clôture et clefs », consacré à l'étude des palissades introduites dans les écoles rurales mexicaines qui, longtemps, s'ouvrirent sur l'espace public sans que rien ne manifestât leurs limites. Clôtures rimèrent avec clefs et l'objet devint un symbole des conflits qui opposèrent les autorités locales au pouvoir national qui s'imposa par leur possession. Vient ensuite un article d'Antonio Viñao qui interroge le sens de la localisation des bureaux de la direction au sein des écoles primaires. Des projets panoptiques de la fin du XIX $\mathrm{X}^{\mathrm{e}}$ siècle où ils étaient placés au centre de l'édifice, permettant une surveillance de l'ensemble des classes rayonnantes, à ceux des années 1920 où ils étaient à l'entrée de l'école, leur situation révèle un rôle conçu de façon plus administrative que pédagogique. Après l'architecture, deux articles sont consacrés aux objets, ceux de Pedro L. Moreno Martinez et d'Inés Dussel. Le premier dresse une histoire du banc-table en Espagne de 1838 à 1936 ; le second, celle des guardapolvos, ces tabliers blancs si répandus dans les écoles d'Argentine que l'on y appelle les écoliers "petites colombes blanches". Introduits dans les établissements religieux par imitation des soutanes, ils furent maintenus par hygiénisme et décence, puis portés par un discours nationaliste et esthétique avant d'incarner, pour les émigrants en particulier, un sentiment de citoyenneté.

4 La réflexion de Catherine Burke sur la lumière comme métaphore et matérialité de l'espace scolaire constitue une sorte d'intermède autour d'une idée séduisante. Elle donne lieu à des hypothèses stimulantes comme celle d'une histoire de l'architecture scolaire à concevoir comme une histoire de fenêtres. Aussi regrettera-t-on qu'elle ne soit pas étayé par une connaissance des travaux en ce domaine qui aurait enrichi l'évocation du plein air ou évité d'apposer le prénom de Frank au pseudonyme de Le Corbusier... Les quatre articles suivants traitent de la conception des objets utilisés dans les classes, de leur vulgarisation et de leur emploi dans l'enseignement. Martin Lawn évoque l'apparition du matériel didactique, son développement, son rangement et sa conservation. Ian Grosvenor analyse les expositions qui ont contribué à sa diffusion et au perfectionnement de son design. Au centre de son propos figure celle de Londres en 1937, « Design in Education », pour laquelle furent mobilisées des personnalités telles que Frank Pick, les architectes Walter Gropius et Maxwell Fry... L'ensemble s'achève par les contributions de Margaret H. White, Carey Jewitt \& Ken Jones qui analysent des pratiques pédagogiques: l'usage du papier et du dessin dans l'enseignement de l'art, celui du temps et de l'espace dans l'apprentissage de l'anglais.

On le voit, les approches sont nombreuses et variées. Dans leur introduction, Martin Lawn et Ian Grosvenor en proposent une généalogie, les situant entre la micro-histoire et la culture matérielle. "The general approach to the study of schooling which we began 
some years ago was expérimental. Although a focused approach to historiography and methodology was developed, it began with images of schooling and then continued with using artefacts [...] Initially we described this as "micro-histories in material culture of education" ${ }^{2}$ "; «L'approche globale de l'étude de l'enseignement avec laquelle nous avons débuté, il y a quelques années, était expérimentale. Bien que nous ayons développé une approche centrée sur l'historiographie et la méthodologie, nous avons commencé avec des images de l'enseignement, et continué en utilisant des objets [...] Nous l'avons d'abord décrite comme une "micro-histoire de la culture matérielle de l'éducation" ». Ils renvoyaient alors aux travaux de Carlo Ginzburg ou d'Emmanuel Le Roy-Ladurie ${ }^{3}$ Cependant, cette façon d'utiliser un document pour expliquer la mentalité d'une époque n'était pas exactement ce que ces derniers souhaitaient faire, se situant plus volontiers dans le sillage de Foucault, en considérant les individus comme gouvernés par les objets, les relations, les espaces... Aussi considèrent-ils aujourd'hui le terme de «culture matérielle» préférable, même s'il n'est pas encore en parfaite adéquation avec leur projet.

Cette nouvelle approche de l'histoire de l'éducation se cherche donc et sa quête est captivante comme le démontre cet ensemble de contributions auquel on ne reprochera que la pauvreté et la médiocre qualité de l'iconographie. Les auteurs ne semblent pas y avoir apporté l'attention qu'exige un regard qui devrait les considérer comme une source et non un plaisir accessoire. Ainsi la couverture, une curieuse photo de fillettes assises en plein air sur la terrasse de ce qui est probablement une école de plein air anglaise, cousant sur d'étranges chaises longues glissées sous des pupitres traditionnels, aurait méritée un commentaire ou tout au moins une légende. Pour l'historienne de l'architecture que je suis, habituée à travailler avec un matériel figuré photos, gravures, dessins, plans... - riche d'informations que ne livre aucun texte, il y a là des ressources qu'il est aussi essentiel de sortir des musées que les objets pour construire cette histoire de l'éducation que les auteurs appellent de leurs vœux.

\section{NOTES}

1. Grosvenor, M. Lawn \& K. Rousmaniere (ed.), Silences and Images: The Social History of Classroom. Bern : P. Lang, 1999, 274 p. (History of Schools and Schooling, vol. 7)

2. M. Lawn, I. Grosvenor, «Imagining a oroject : networks, discourses and spaces - towards a new archeology of urban education ». Pcedagogica Historica, 2000, vol. XXV, n 2, p. 381-393.

3. C. Ginzburg, Le fromage et les vers: l'univers d'un meunier du XVI siècle. Paris: Flammarion, 1980 (éd. originale 1976) ; E. Le Roy-Ladurie, Montaillou, village occitan de 1294 à 1324. Paris : Gallimard, 1975. 


\section{AUTEURS}

ANNE-MARIE CHÂTELET

École nationale supérieure d'architecture, Versailles 